\title{
20. GEOCHEMISTRY OF CARBON, DSDP LEG 38
}

\author{
J.G. Erdman and K.S. Schorno, Phillips Petroleum Company, Bartlesville, Oklahoma
}

\section{INTRODUCTION}

A total of 42 frozen core samples from the North Sea, DSDP Leg 38, was received through the JOIDES Advisory Panel on Organic Geochemistry. These samples came from Sites 338, 339, 341, 344, 346, 348, and 349 (Figure 1). For Sites 338, 339, and 341 on the $\mathrm{V} \phi$ ring Plateau and for Site 344 southwest of Spitzbergen, the samples studied represented the full suite of frozen cores taken for organic geochemical study. Thus, for these sites the data generated provide maximal information in regard to trends with depth. In the case of Site 338, lateral uniformity at varying present depths of burial, but equivalent geologic age, was evaluated through the study of samples east of the ridge where the sedimentary section is thicker.

All frozen core samples for this study and other organic geochemical studies performed were taken and preserved under the personal shipboard supervision of David A. Morris of this laboratory.

\section{Sampling and Study Procedures}

The core samples were collected in their plastic liners, frozen onboard ship, and maintained frozen thereafter.

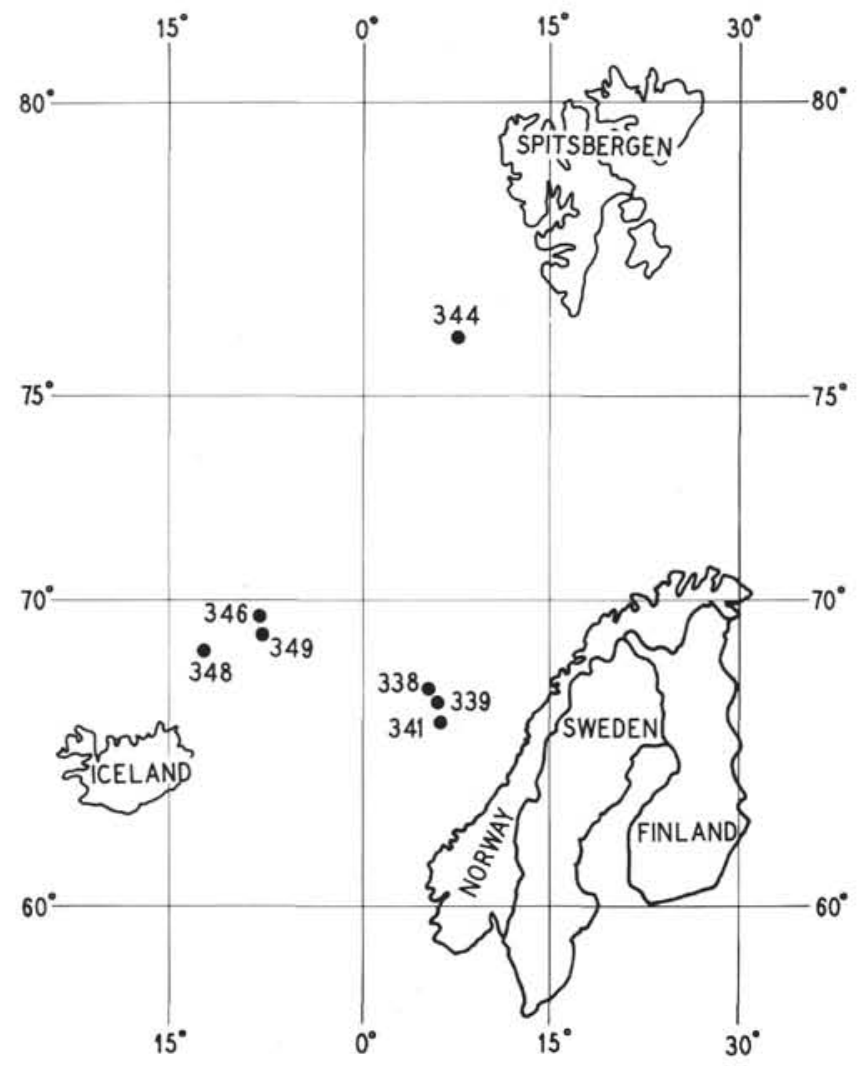

Figure 1. Location of sites from which samples for geochemical study were obtained.
Upon reaching the laboratory, the frozen samples were split for characterization and distribution among laboratories participating in the study.

The flat frozen surface of one longitudinal half of each core section was smoothed for description and photographing using a milling machine and flycutter. The faces of the samples were then allowed to thaw just sufficiently to eliminate frost. Depth of thawing usually was no more than 1 to $2 \mathrm{~mm}$.

Each sample was photographed both in color and black and white and described. In each photograph a metric scale and a color or density standard were included for matching during photographic printing to ensure accurate reproduction of density values. Although all 42 cores were photographed, only 16 samples (Figures 2-16) are included due to space limitations. Descriptions of the lithologies are given in Table 1. Provided also in Table 1 are the authors' opinion of the degree of disturbance of those cores for which photographs are not shown. During the description 10 to $20 \mathrm{mg}$ samples of each lithology were removed with a spatula for determination of carbonate carbon and organic carbon (Figures 2-16 and Table 1). Finally, the core halves were hard frozen and split from top to bottom to provide further aliquots for chemical study and archiving. Those slices intended for immediate study in this laboratory were cleaned on all exposed surfaces.

A flow diagram for separating and determining carbon species is shown in Figure 17. The solvents were distilled in glass immediately before use. Each analytical determination was performed in duplicate.

\section{Core Characterization}

Organic geochemistry is largely a study of changes in chemical composition of organic matter incorporated in sediments as a function of five variables: namely (1) the concentration of organic matter in the source rock, (2) the geologic time interval (age), (3) the thermal history (temperature), (4) the extent of abiogenic oxidation, and (5) the mineral matrix (Erdman, 1975a, b). For this reason, emphasis was placed on studying all samples taken from top to bottom in holes or groups of holes. Therefore, the samples permit evaluation of the maximum effect of variations in geologic age, present and past depths of burial, and initial environment of deposition. Furthermore, an assessment of the reliability to be placed on each sample is considered essential to acceptance of the data.

Photographs and core descriptions provided in Table 1 and Figures 2 through 16 show wide variations both in deposition conditions and in texture, and to some extent, the chemical composition of the mineral matrix. These photographs further show that, in some cases, there has been extensive disturbance and mixing as a consequence of drilling. In Sections 339-8-4, 339-12-1, 


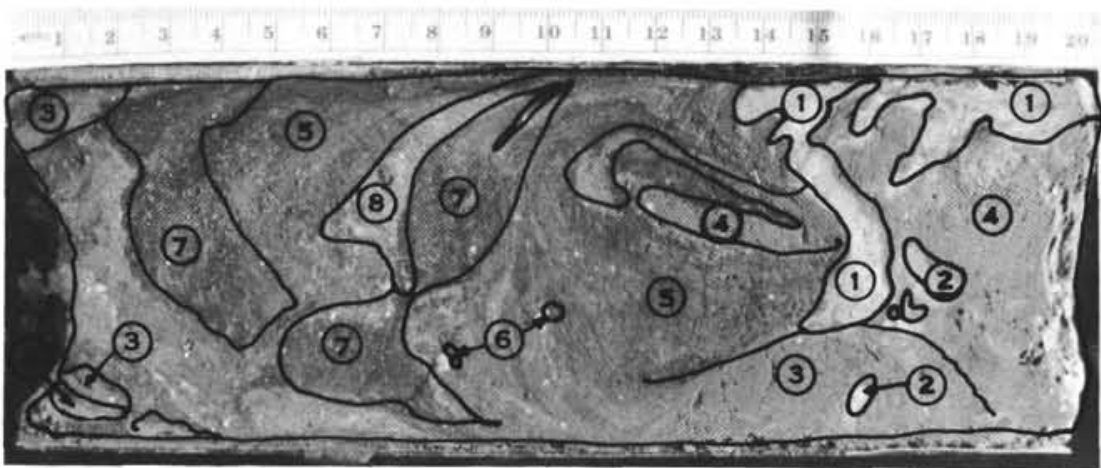

Figure 2. Site 339, Core 8, Section 4, (1) Clay, light olive gray (5Y 5/2), plastic, apparently pelleted. (2) Clay, grayish-olive (10Y 4/2), plastic. (3) Clay, dusky yellowish-brown (10Y R 2/2), Plastic, silty, calcareous. Common Foram tests. (4) Clay, olive gray (5Y 3/2), plastic, silty, calcareous. Common foram tests. Interlaminated on a 1-mm scale with (5). (5) Clay, as (d), but dark greenish gray (5GY 4/1) (6) Pebble, granite (7) Clay, as (4), but noncalcareous. No foram tests, sand and pebbles of igneous/metamorphic rock. (8) Clay, as (7), but slightly lighter in color olive gray, $5 Y$ 4/2.1

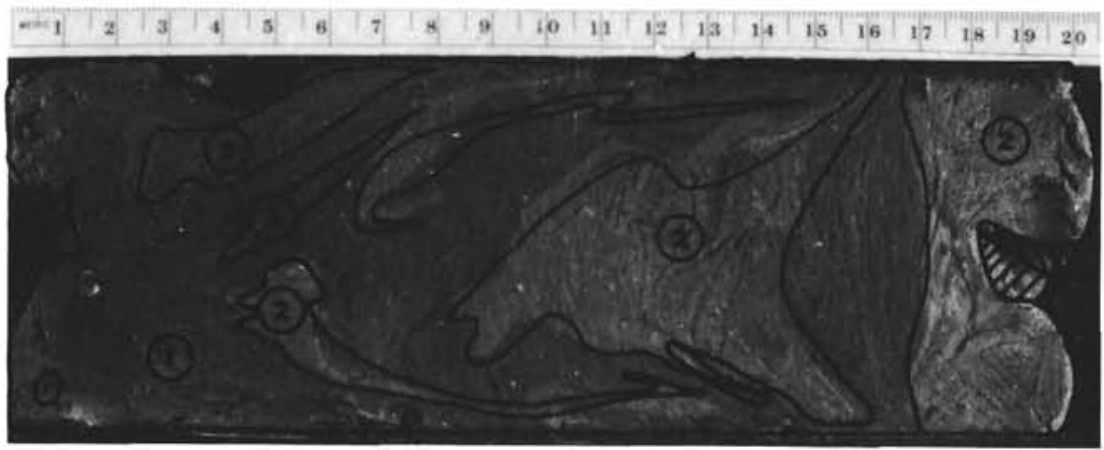

Figure 4. Site 341, Core 4, Section 1. (1) Clay, olive black (5Y 2/1) with scattered quartz sand to $2 \mathrm{~mm} .1 \mathrm{~cm}$. brown sparry calcite module. (2) Clay, greenish-gray (5G 6/1) with scattered quartz sand to $2 \mathrm{~mm}$. (3) Clay, olive-gray $(5 Y 4 / 2)$ with scattered quartz sand to $2 \mathrm{~mm}$.

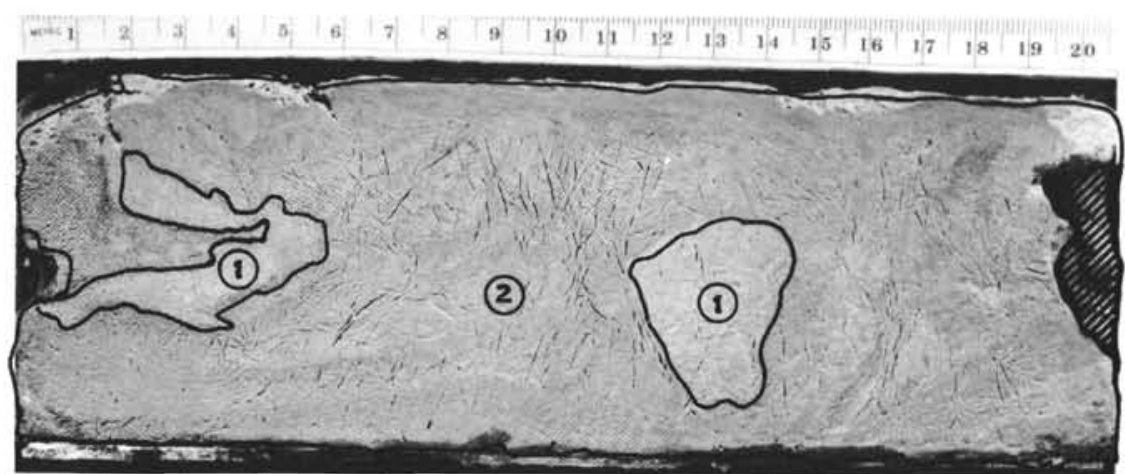

Figure 3. Site 339, Core 12, Section 1. (1) Clay, light olive gray (5Y 5/2) plastic, appears pelleted (2) same as above, but olive gray (5Y 4/1).

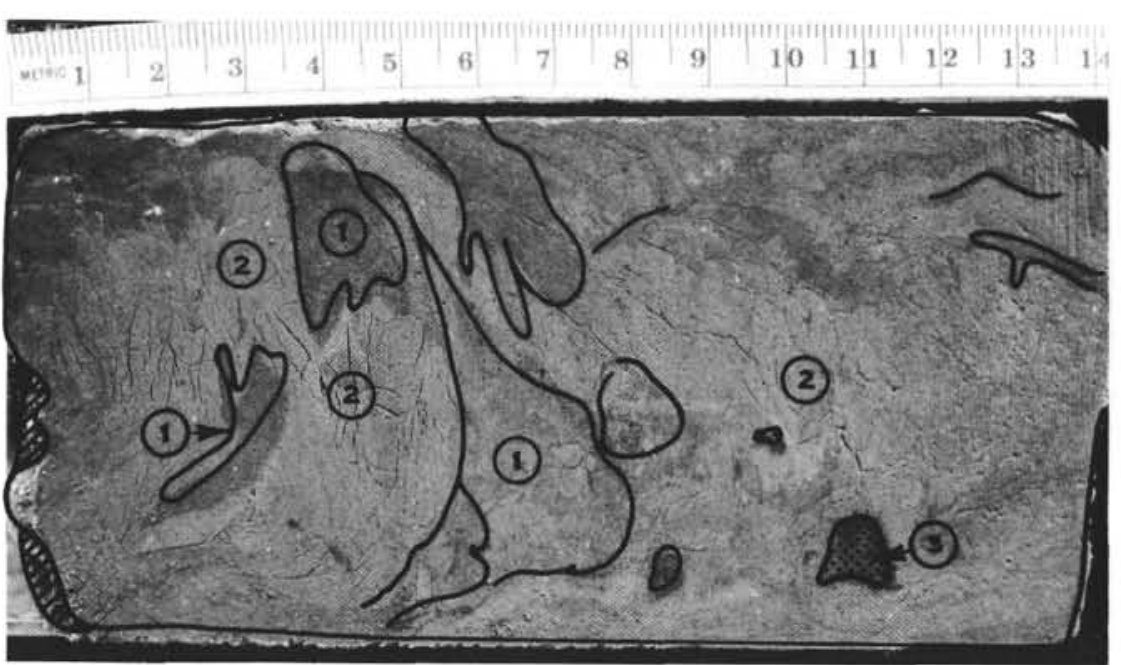

Figure 5. Site 346, Core 12, Section 4. (1) Silt and clay, olive-gray (5Y 3/2). Muscovite and angular to subrounded quartz. Acicular microfossils (diatoms, spicules?). (2) Silt and clay as above, but light olive-gray (5Y 3/2). (3) Olive-gray (5Y 3/2) dense cemented module of pyrite (3). 


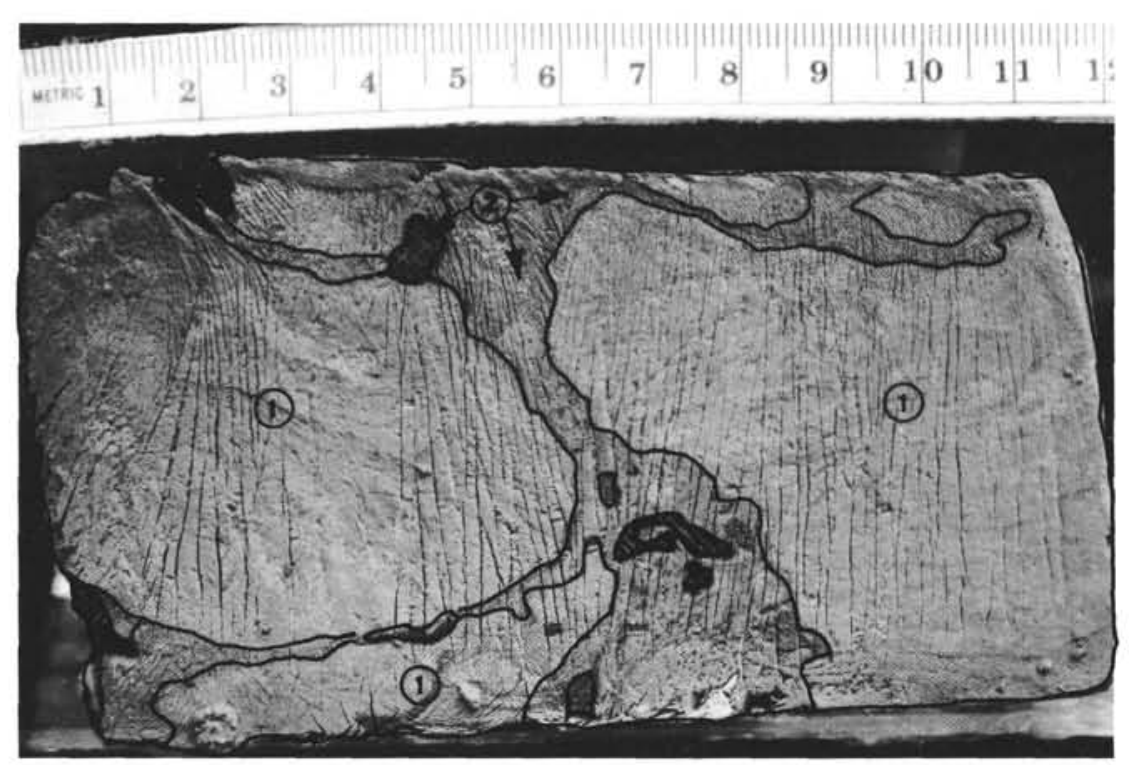

Figure 6. Site 338, Core 11, Section 2. (1) Clay, grayish-olive (10Y 4/2), plastic. Contains scattered silt-sized glauconite, indistinct laminae suggest rotation of blocks within core barrel. (2) Clay, as above but olive-gray (5Y 3/2).

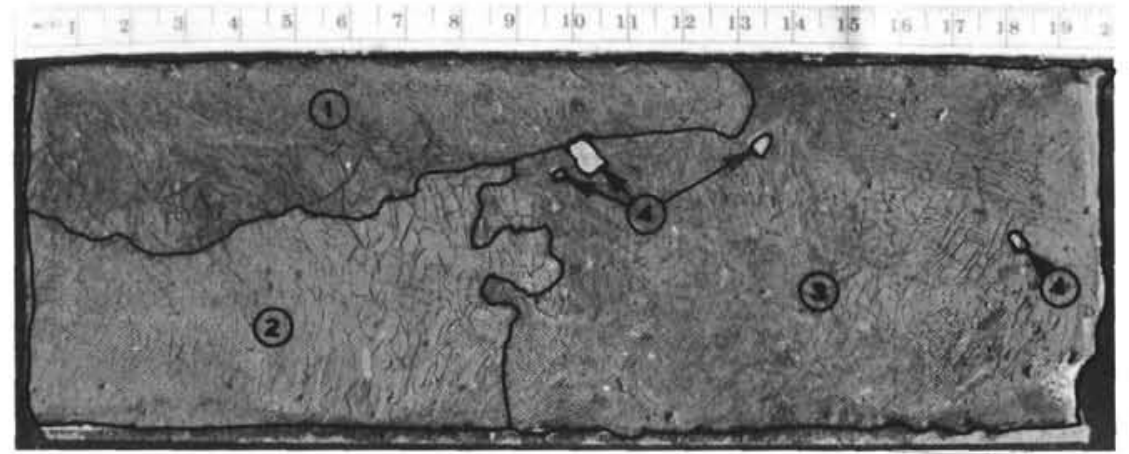

Figure 8. Site 344, Core 5, Section 5. (1) Clay, olive-gray (5Y 3/2). plastic
(2) Clay, as in (a), but olive-gray (5Y 4/1) (3) Clay, olive-black (5Y 2/1). silty, plastic. (4) Sandstone pebble, rounded quartz sand to $500 \mu$ in.

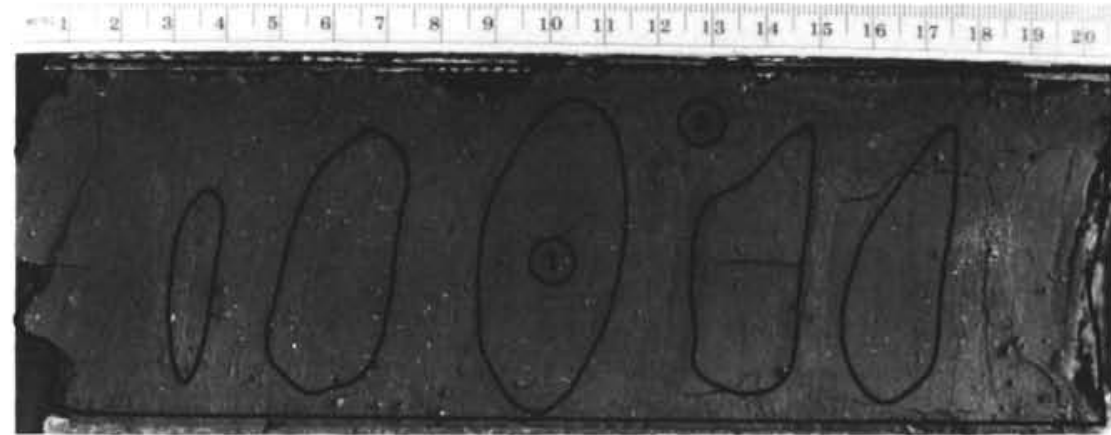

Figure 7. Site 341, Core 32, Section 5. (a) Clay, dusky yellow (5Y 6/4), stiff, apparently laminated in 5-8 $\mathrm{mm}$ layers. Laminations do not extend into Zone 2. (b) Clay, olive-gray (5Y 3/2). Softer than Zone 1 and apparently structureless.

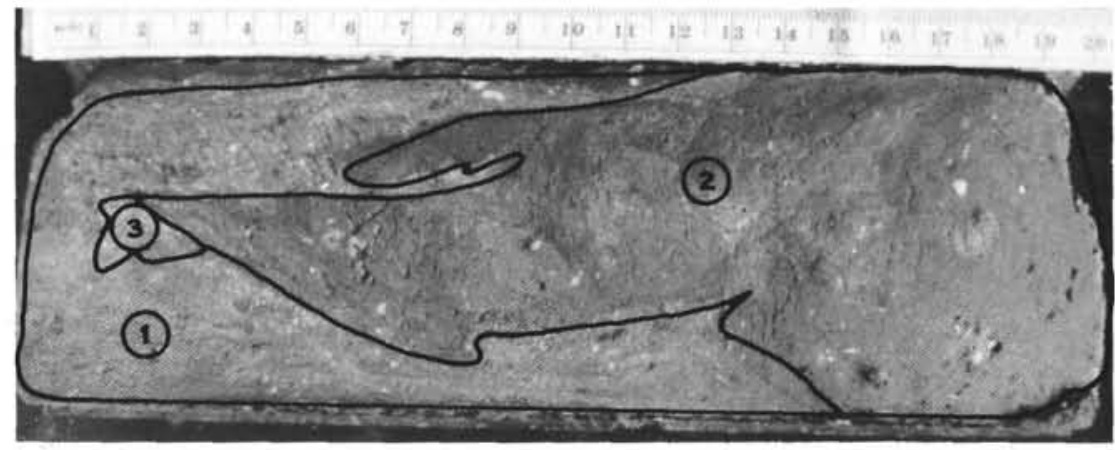

Figure 9. Site 344, Core 9, Section 5. (1) Clay, dark greenish-gray (5G Y 4/1). with coarse sand - to small pebbles - (approx. 2 to $8 \mathrm{~mm}$ ) sized quartz and feldspar, subrounded to angular. (2) Clay, olive-gray (5Y 3/2). 


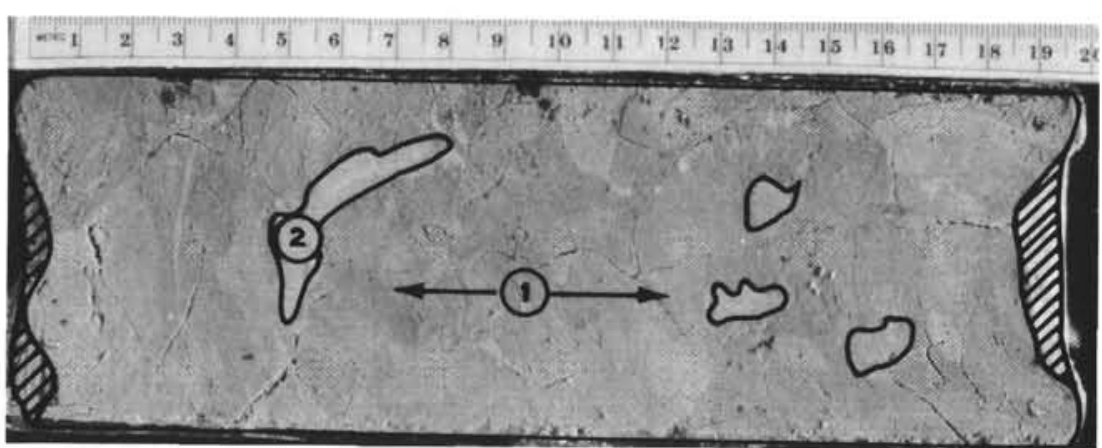

Figure 10. Site 338, Core 26, Section 2. (1) Clay, greenish-gray (5GY 6/1) Apparently structureless. Scattered angular to subrounded quartz silt. (2) Same as above but light greenish-gray (5G 8/1).

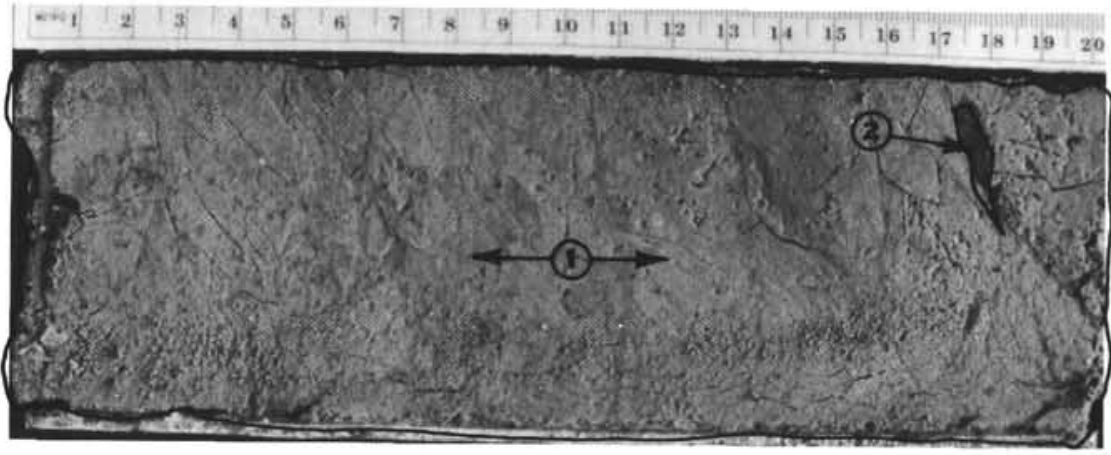

Figure 11. Site 338, Core 19, Section 4. (1) Clay, grayish-olive (10Y 4/2), plastic. Apparently pelleted. Contains scattered quartz silt and acicular microfossils (spicules?). (2) Clay, olive-black (5Y 2/1), plastic.

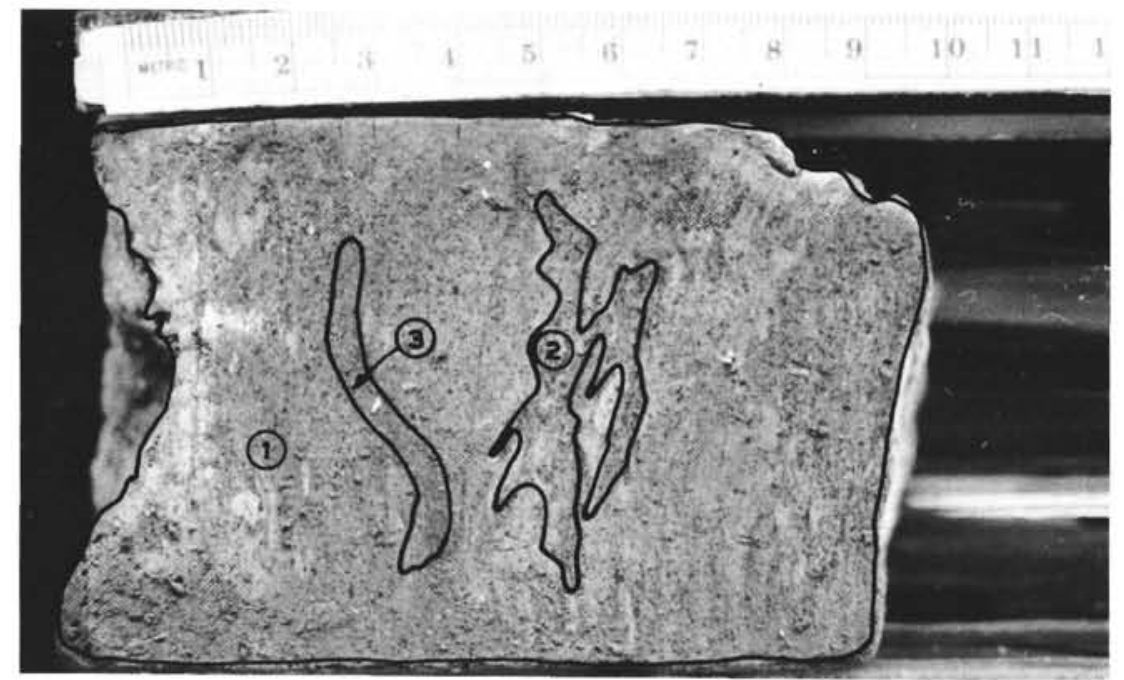

Figure 12. Site 338, Core 30, Section 5. (1) Clay, olive-gray (5Y 3/2), plastic, silty. Contains very abundant $0.5 \mathrm{~mm}$ rounded grains of glauconite. Mottled with patches of light olive-gray (5Y 5/1. (2) Sand, grayish olive-green (5GY 3/2) glauconitic. Rounded 0.5-1.5 mm grains, Friable. (3) Clay, dark yellowish-brown (10YR 3/2), plastic, abundant $0.5 \mathrm{~mm}$ glauconite grains.

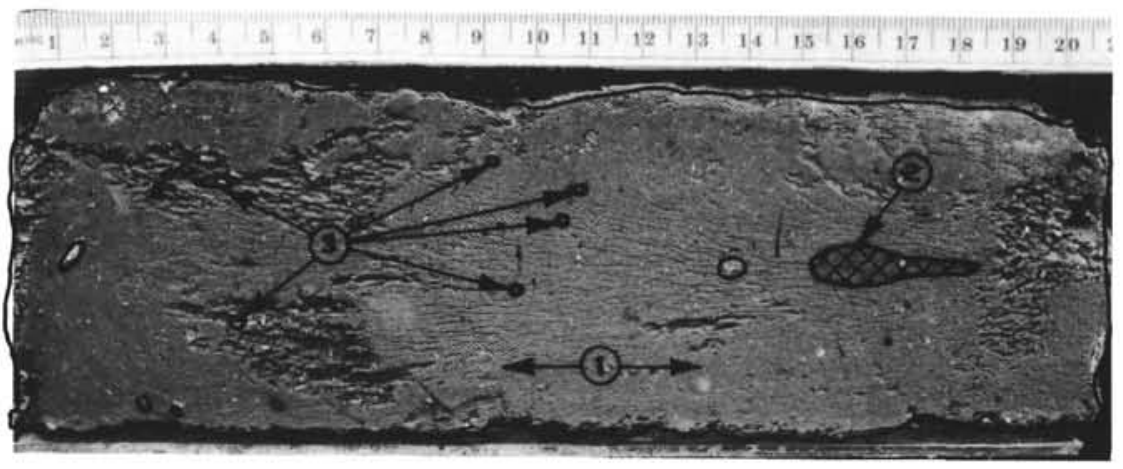

Figure 13. Site 339, Core 4, Section 2. (1) Clay, olive gray (5Y 3/2), plastic, calcareous. (2) Clay, olive-black (5Y 2/1), plastic. (3) Metamorphic rock fragments, $1-8 \mathrm{~mm}$. 


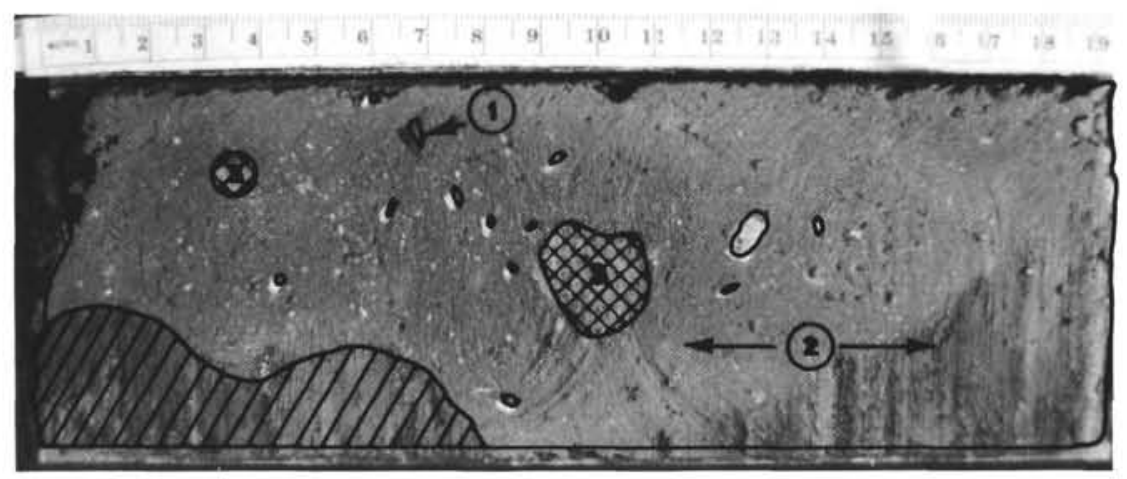

Figure 14. Site 341, Core 10, Section 5. (1) Clay dark greenish-gray (5GY 4/1). Apparently structureless. (2) Scattered silt, sand, and pebbles of igneous/metamorphic rock to $2 \mathrm{~cm}$. (3) Occasional $1 \mathrm{~mm}$ grains of pyrite alternating to limonite.

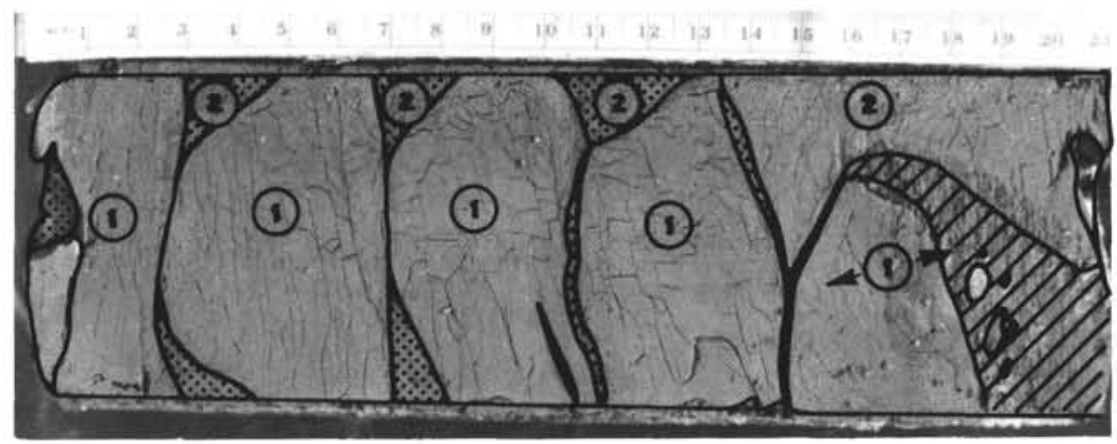

Figure 15. Site 341, Core 30, Section 5. (1) Clay, olive-black (5Y 2/1), with scattered quartz silt and sand to $1-\mathrm{mm}$. Indistinct laminations. (2) Same as above, but contorted and homogenized.

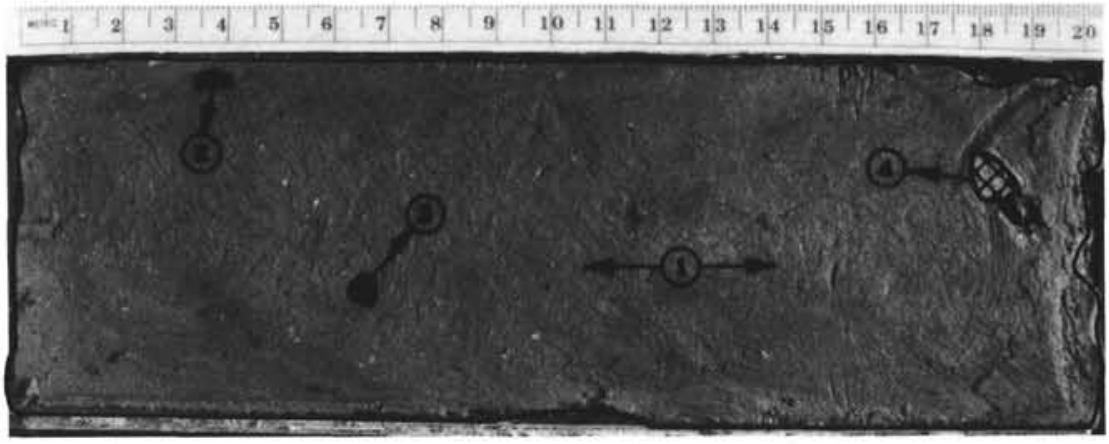

Figure 16. Site 344, Core 7, Section 5. (1) Clay, olive-gray (5Y 3/2). plastic. Contains abundant quartz grains and metamorphic and sedimentary pebbles 1-10 mm. (2) Clay, as in (a), but black (N1). (3) Pebble, shale. (4) Pebble, pelitic schist. 
TABLE 1

Lithological Discriptions and Carbonate and Organic Carbon Values in Weight Percent for Lithologies in Core Sections

\begin{tabular}{|c|c|c|c|c|}
\hline \multirow[b]{2}{*}{ Section } & \multirow[b]{2}{*}{ Lithological Description } & \multicolumn{2}{|c|}{ Carbonate As } & \multirow{2}{*}{$\begin{array}{l}\text { Organi } \\
\text { Carbor }\end{array}$} \\
\hline & & Carbon & $\mathrm{CaCO}_{3}$ & \\
\hline & $\begin{array}{l}\text { Extensive disturbance } \\
\text { throughout core }\end{array}$ & & & \\
\hline \multirow[t]{8}{*}{$339-8-4$} & 1. See Figure 2 & 0.26 & 2.17 & 0.63 \\
\hline & 2. See Figure 2 & 0.19 & 1.58 & 1.10 \\
\hline & 3. See Figure 2 & 1.57 & 13.08 & 0.59 \\
\hline & 4. See Figure 2 & 0.71 & 5.92 & 0.37 \\
\hline & 5. See Figure 2 & 0.03 & 0.25 & 0.28 \\
\hline & 6. See Figure 2 & 0.00 & 0.00 & 0.00 \\
\hline & 7. See Figure 2 & 0.01 & 0.08 & 0.66 \\
\hline & 8. See Figure 2 & 0.06 & 0.50 & 0.03 \\
\hline \multirow[t]{2}{*}{$339-12-1$} & 1. See Figure 3 & 0.24 & 2.00 & 0.81 \\
\hline & 2. See Figure 3 & 0.15 & 1.25 & 0.75 \\
\hline \multirow{3}{*}{$341-4-1$} & 1. See Figure 4 & 0.16 & 1.33 & 0.55 \\
\hline & 2. See Figure 4 & 0.87 & 7.25 & 0.33 \\
\hline & 3. See Figure 4 & - & - & - \\
\hline \multirow[t]{4}{*}{$346-12-4$} & 1. See Figure 5 & 0.10 & 0.83 & 1.36 \\
\hline & 2. See Figure 5 & 0.08 & 0.67 & 1.02 \\
\hline & 3. See Figure 5 & 0.00 & 0.00 & 0.00 \\
\hline & $\begin{array}{l}\text { Lumps of undisturbed } \\
\text { core }\end{array}$ & & & \\
\hline \multirow[t]{2}{*}{$338-11-2$} & 1. See Figure 6 & 0.00 & 0.00 & 0.78 \\
\hline & 2. See Figure 6 & 0.00 & 0.00 & 1.27 \\
\hline \multirow[t]{4}{*}{$341-20-3$} & $\begin{array}{l}\text { 1. Clay, olive-gray (5Y } \\
4 / 2) \text {, with angular to } \\
\text { subround quartz to } \\
1 \mathrm{~mm} \text { scattered to } \\
\text { rare; indistinct to con- } \\
\text { tinuous laminations }\end{array}$ & 2.06 & 17.17 & 0.36 \\
\hline & $\begin{array}{l}\text { 2. As in 1, but contorted } \\
\text { and homogenized }\end{array}$ & 2.10 & 17.50 & 0.36 \\
\hline & $\begin{array}{l}\text { 3. Clay, brownish-gray } \\
(5 \mathrm{Y} 4 / 1) \text {, as in } 1\end{array}$ & 0.69 & 5.75 & 0.40 \\
\hline & $\begin{array}{l}\text { 4. Clay, olive-gray ( } 5 \mathrm{Y} \\
3 / 2 \text { ), as in } 1 \text {, but } \\
\text { with quartz to } 4 \mathrm{~mm} \\
\text { diameter }\end{array}$ & 1.23 & 10.25 & 0.40 \\
\hline \multirow[t]{2}{*}{$341-32-5$} & 1. See Figure 7 & 0.17 & 1.42 & 1.69 \\
\hline & 2. See Figure 7 & 0.17 & 1.42 & 1.65 \\
\hline \multirow[t]{3}{*}{$344-5-5$} & 1. See Figure 8 & 0.05 & 0.42 & 0.70 \\
\hline & 2. See Figure 8 & 0.10 & 0.83 & 0.47 \\
\hline & 3. See Figure 8 & 0.18 & 1.50 & 0.78 \\
\hline \multirow[t]{4}{*}{$344-9-5$} & 4. See Figure 8 & $\begin{array}{l}0.00 \\
0.18\end{array}$ & $\begin{array}{l}0.00 \\
1.50\end{array}$ & $\begin{array}{l}0.00 \\
0.19\end{array}$ \\
\hline & 2. See Figure 9 & 0.08 & 0.67 & 0.47 \\
\hline & 3. See Figure 9 & 0.18 & 1.50 & 0.22 \\
\hline & $\begin{array}{l}\text { Questionably disturbed } \\
\text { core }\end{array}$ & & & \\
\hline \multirow[t]{2}{*}{$338-8-2$} & $\begin{array}{l}\text { 1. Clay, olive-black ( } 5 \mathrm{Y} \\
2 / 1) \text {, plastic; contains } \\
1 \mathrm{~mm} \text { and larger frag- } \\
\text { ments of } 2 \text { below }\end{array}$ & 0.30 & 2.50 & 0.58 \\
\hline & $\begin{array}{l}\text { 2. Clay, as above, but } \\
\text { grayish-olive ( } 5 \mathrm{GY} \\
3 / 2)\end{array}$ & 0.03 & 0.25 & 1.53 \\
\hline $338-22-5$ & $\begin{array}{l}\text { 1. Clay, greenish-gray } \\
\text { (5QY 6/1), plastic, } \\
\text { mottled to indistinctly } \\
\text { interbedded with } 2 \\
\text { below. Calcareous } \\
\text { clay contains discon- } \\
\text { tinuous inclusions of } \\
3 \text { below, some along } \\
\text { apparent bedding } \\
\text { surfaces }\end{array}$ & 0.31 & 2.58 & 0.04 \\
\hline
\end{tabular}

TABLE 1 - Continued

\begin{tabular}{|c|c|c|c|c|}
\hline \multirow[b]{2}{*}{ Section } & \multirow[b]{2}{*}{ Lithological Description } & \multicolumn{2}{|c|}{ Carbonate As } & \multirow{2}{*}{$\begin{array}{l}\text { Organic } \\
\text { Carbon }\end{array}$} \\
\hline & & Carbon & $\mathrm{CaCO}_{3}$ & \\
\hline & $\begin{array}{l}\text { 2. Clay, as above, but } \\
\text { greenish-gray }(5 \mathrm{G} 6 / 1)\end{array}$ & 3.13 & 26.08 & 0.23 \\
\hline & $\begin{array}{l}\text { 3. Friable agglomerations } \\
\text { of } 100-200 \mu \mathrm{m} \text { euhe- } \\
\text { dral pyrite }\end{array}$ & 0.00 & 0.00 & 0.00 \\
\hline \multirow[t]{2}{*}{$338-26-2$} & 1. See Figure 10 & 0.02 & 0.17 & 0.13 \\
\hline & 2. See Figure 10 & 0.00 & 0.00 & 0.30 \\
\hline \multirow[t]{2}{*}{$339-6-2$} & $\begin{array}{l}\text { 1. Clay, olive-gray ( } 5 \mathrm{Y} \\
3 / 2 \text { ), plastic, struc- } \\
\text { tureless, slightly cal- } \\
\text { careous, scattered } \\
\text { rounded quartz grains } \\
\text { to } 250 \mu \mathrm{m} \text { rare pyrite } \\
\text { nodules to } 250 \mu \mathrm{m}\end{array}$ & 0.03 & 0.25 & 1.12 \\
\hline & $\begin{array}{l}\text { 2. Clay, grayish-olive } \\
\text { (10Y 4/2), plastic } \\
\text { structureless }\end{array}$ & 0.04 & 0.33 & 0.48 \\
\hline \multirow[t]{2}{*}{$341-6-2$} & $\begin{array}{l}\text { 1. Clay, olive-gray ( } 5 \mathrm{Y} \\
3 / 2 \text { ) with scattered } \\
\text { quartz sand and clasts } \\
\text { of } 2 \text { below to } 5 \mathrm{~mm}\end{array}$ & 0.66 & 5.50 & 0.56 \\
\hline & $\begin{array}{l}\text { 2. Clay, dusky yellow- } \\
\text { green }(5 G Y 5 / 2) \\
\text { 3. Clay, olive-black } \\
(5 Y 2 / 1)\end{array}$ & 0.40 & 3.30 & 0.47 \\
\hline \multirow[t]{2}{*}{$344-27-3$} & $\begin{array}{l}\text { 1. Silt, olive-gray ( } 5 Y \\
4 / 1 \text { ), firm clayey }\end{array}$ & 0.04 & 0.33 & 0.69 \\
\hline & $\begin{array}{l}\text { 2. Clay, olive-black ( } 5 \mathrm{Y} \\
2 / 1 \text { ), firm to slightly } \\
\text { plastic }\end{array}$ & 0.46 & 3.83 & 1.44 \\
\hline \multirow[t]{3}{*}{$344-31-2$} & $\begin{array}{l}\text { 1. Clay, sandy, olive- } \\
\text { black }(5 Y 2 / 1) \text {, firm } \\
\text { slightly calcareous, } \\
\text { interlaminated on a } \\
2-5 \mathrm{~mm} \text { scale with } 2\end{array}$ & 0.29 & 2.42 & 0.65 \\
\hline & $\begin{array}{l}\text { 2. Sand, light olive-gray } \\
\text { ( } 5 \text { Y } 5 / 2) \text { friable to } \\
\text { dense, carbonate } \\
\text { cement; some lami- } \\
\text { nations are silt and } \\
\text { clay rich }\end{array}$ & 0.19 & 1.58 & 0.52 \\
\hline & Undisturbed core & & & \\
\hline $338-5-5$ & $\begin{array}{l}\text { 1. Silt and clay, olive- } \\
\text { gray }(5 \mathrm{Y} 4 / 1) \text {; ap- } \\
\text { parently structureless }\end{array}$ & 0.13 & 1.08 & 2.87 \\
\hline \multirow[t]{2}{*}{$338-13-5$} & $\begin{array}{l}\text { 1. Clay, light olive-gray } \\
\text { (5Y 5/2), plastic; } \\
\text { contains silt appar- } \\
\text { ently structureless }\end{array}$ & 0.07 & 0.58 & 1.42 \\
\hline & $\begin{array}{l}\text { 2. Clay, olive-black } \\
(5 \mathrm{Y} 2 / 1)\end{array}$ & 0.06 & 0.50 & 1.32 \\
\hline $338-17-5$ & $\begin{array}{l}\text { 1. Silt, light olive-gray } \\
\text { (5Y 5/2) primarily } \\
\text { quartz, some clay, } \\
\text { firm, apparently } \\
\text { structureless }\end{array}$ & 0.00 & 0.00 & 1.55 \\
\hline \multirow[t]{2}{*}{$338-19-4$} & 1. See Figure 11 & 0.00 & 0.00 & 0.57 \\
\hline & 2. See Figure 11 & 0.08 & 0.67 & 1.42 \\
\hline $338-24-5$ & $\begin{array}{l}\text { 1. Clay, grayish-green } \\
(10 \mathrm{GY} 5 / 2) \text { appar- } \\
\text { ently structureless }\end{array}$ & 0.15 & 1.25 & 0.17 \\
\hline \multirow[t]{3}{*}{$338-30-5$} & 1. See Figure 12 & 0.00 & 0.00 & 0.02 \\
\hline & 2. See Figure 12 & 0.05 & 0.42 & 0.24 \\
\hline & 3. See Figure 12 & - & - & - \\
\hline
\end{tabular}


TABLE 1 - Continued

\begin{tabular}{|c|c|c|c|c|}
\hline \multirow[b]{2}{*}{ Section } & \multirow[b]{2}{*}{ Lithological Description } & \multicolumn{2}{|c|}{ Carbonate As } & \multirow{2}{*}{$\begin{array}{l}\text { Organic } \\
\text { Carbon }\end{array}$} \\
\hline & & Carbon & $\mathrm{CaCO}_{3}$ & \\
\hline $338-32-3$ & $\begin{array}{l}\text { 1. Clay, olive-gray (5Y } \\
3 / 2 \text { ), plastic, appar- } \\
\text { ently structureless }\end{array}$ & 0.20 & 1.67 & 0.44 \\
\hline \multirow[t]{3}{*}{$339-4-2$} & 1. See Figure 13 & 1.25 & 10.42 & 0.62 \\
\hline & 2. See Figure 13 & 0.56 & 4.67 & 0.51 \\
\hline & 3. See Figure 13 & 0.79 & 6.58 & 0.90 \\
\hline \multirow[t]{3}{*}{$341-2-1$} & $\begin{array}{l}\text { 1. Clay, olive-gray } \\
(5 Y 3 / 2) \text {, with silt } \\
\text { and rare quartz } \\
\text { pebbles to } 5 \mathrm{~mm}\end{array}$ & 0.78 & 6.50 & 0.30 \\
\hline & $\begin{array}{l}\text { 2. Clay, olive-black ( } 5 \mathrm{Y} \\
2 / 1) \text {, plastic, struc- } \\
\text { tureless }\end{array}$ & 0.31 & 2.58 & 0.03 \\
\hline & $\begin{array}{l}\text { 3. Same as } 2 \text { above but } \\
\text { black }\left(\mathrm{N}_{1}\right)\end{array}$ & 0.34 & 2.83 & 0.72 \\
\hline $341-8-3$ & $\begin{array}{l}\text { 1. Clay, dark greenish- } \\
\text { gray }(5 \mathrm{GH} 4 / 1) \text { with } \\
\text { subangular to rounded } \\
\text { quartz silt, sand and } \\
\text { igneous } / \text { metamorphic } \\
\text { pebbles to } 1 \mathrm{~cm}\end{array}$ & 0.60 & 5.00 & 0.58 \\
\hline & $\begin{array}{l}\text { 2. Same as above but } \\
\text { olive-black }(5 \text { Y } 2 / 1)\end{array}$ & 0.06 & 0.50 & 0.18 \\
\hline \multirow[t]{3}{*}{$341-10-5$} & 1. See Figure 14 & 1.10 & 9.20 & 0.45 \\
\hline & 2. See Figure 14 & 0.92 & 7.67 & 0.43 \\
\hline & 3. See Figure 14 & 0.00 & 0.00 & 0.00 \\
\hline $341-23-4$ & $\begin{array}{l}\text { 1. Clay, olive-gray (5Y } \\
\text { 4/1) with rare quartz } \\
\text { sand, common silt, } \\
\text { structureless to in- } \\
\text { distinctly laminated }\end{array}$ & 0.08 & 0.67 & 0.48 \\
\hline \multirow[t]{2}{*}{$341-25-5$} & $\begin{array}{l}\text { 1. Clay, olive-gray (5Y } \\
\text { 3/2) silty, slightly } \\
\text { calcareous, firm; in- } \\
\text { distinctly laminated }\end{array}$ & 0.06 & 0.50 & 1.22 \\
\hline & $\begin{array}{l}\text { 2. Clay, as above, but } \\
\text { plastic and deformed }\end{array}$ & 0.04 & 0.33 & 1.22 \\
\hline \multirow[t]{2}{*}{$341-28-5$} & $\begin{array}{l}\text { 1. Clay, olive-gray ( } 5 \mathrm{Y} \\
3 / 2) \text {, structureless to } \\
\text { indistinctly laminated }\end{array}$ & 0.93 & 7.75 & 0.87 \\
\hline & $\begin{array}{l}\text { 2. Same as above but } \\
\text { olive-black }(5 Y 2 / 1)\end{array}$ & 0.81 & 6.75 & 0.87 \\
\hline \multirow[t]{2}{*}{$341-30-5$} & 1. See Figure 15 & 0.01 & 0.08 & 0.23 \\
\hline & 2. See Figure 15 & 0.08 & 0.67 & 1.19 \\
\hline $341-34-5$ & $\begin{array}{l}\text { 1. Clay, olive-gray ( } 5 Y \\
3 / 2 \text { ), plastic to firm, } \\
\text { scattered quartz silt }\end{array}$ & 0.03 & 0.25 & 1.48 \\
\hline \multirow[t]{4}{*}{$344-7-5$} & 1. See Figure 16 & 0.12 & 1.00 & 0.27 \\
\hline & 2. See Figure 16 & 0.06 & 0.50 & 0.82 \\
\hline & 3. See Figure 16 & - & - & - \\
\hline & 4. See Figure 16 & - & - & - \\
\hline $344-11-1$ & $\begin{array}{l}\text { 1. Clay, olive-gray (5Y } \\
3 / 2 \text { ), plastic, contains } \\
5-10 \mathrm{~mm} \text { pyrite } \\
\text { nodules }\end{array}$ & 0.75 & 6.25 & 1.44 \\
\hline & $\begin{array}{l}\text { 2. Clay, dark greenish- } \\
\text { gray }(5 \mathrm{GY} 4 / 1) \text {, plas- } \\
\text { tic, contains pyrite } \\
\text { nodules as in } 1\end{array}$ & 0.05 & 0.42 & 0.51 \\
\hline & 3. Sandstone pebble & 1.77 & 14.75 & 1.22 \\
\hline $344-15-4$ & $\begin{array}{l}\text { 1. Clay, silty, olive-gray } \\
(5 Y 3 / 2) \text { plastic, } \\
\text { structureless } \\
\text { 2. Sandstone pebble }\end{array}$ & 0.17 & 1.42 & 1.11 \\
\hline $346-1-3$ & $\begin{array}{l}\text { 1. Clay, dark yellowish- } \\
\text { brown (10 YR 4/2), } \\
\text { slightly calcareous, } \\
\text { scattered silt to sand- }\end{array}$ & 0.83 & 6.92 & 0.18 \\
\hline
\end{tabular}

TABLE 1 - Continued

\begin{tabular}{|c|c|c|c|c|}
\hline \multirow[b]{2}{*}{ Section } & \multirow{2}{*}{ Lithological Description } & \multicolumn{2}{|c|}{ Carbonate As } & \multirow{2}{*}{$\begin{array}{l}\text { Organic } \\
\text { Carbon }\end{array}$} \\
\hline & & Carbon & $\mathrm{CaCO}_{3}$ & \\
\hline & $\begin{array}{l}\text { size quartz; plastic } \\
\text { structureless }\end{array}$ & & & \\
\hline $346-10-4$ & $\begin{array}{l}\text { 1. Clay, olive-gray (5Y } \\
\text { 3/2), plastic, appears } \\
\text { pelleted; acicular sili- } \\
\text { ceous microfossils } \\
\text { (spicules?) very abun- } \\
\text { dant; } 250-500 \mu \mathrm{m} \\
\text { quartz; muscovite } \\
\text { and pyrite common }\end{array}$ & 0.02 & 0.17 & 1.13 \\
\hline $346-17-2$ & $\begin{array}{l}\text { 1. Silt, olive-gray (5Y } \\
5 / 2) \text {, firm, sandy; } \\
\text { quartz, muscovite, } \\
\text { and clay common, } \\
\text { mottled with more } \\
\text { clay-rich zones }\end{array}$ & 0.05 & 0.42 & 0.43 \\
\hline & $\begin{array}{l}\text { 2. Silt, dark gray (N4), } \\
\text { plastic, very clay-rich } \\
(30 \%-50 \%) \text {; apparen- } \\
\text { tly contorted }\end{array}$ & & & \\
\hline $348-19-5$ & $\begin{array}{l}\text { 1. Clay, silty, grayish- } \\
\text { olive-green (5GY } 3 / 2 \text { ); } \\
\text { apparently structure- } \\
\text { less }\end{array}$ & 0.05 & 0.42 & 0.40 \\
\hline $348-23-5$ & $\begin{array}{l}\text { 1. Clay, olive-gray (5Y } \\
\text { 3/2), plastic, abun- } \\
\text { dant silt-sized quartz } \\
\text { and muscovite; struc- } \\
\text { tureless }\end{array}$ & 0.05 & 0.42 & 0.47 \\
\hline
\end{tabular}

and $346-12-4$, the cores appear to be thoroughly disturbed and mixed (Figures 2-5). In Sections 338-11-2, $341-20-3,341-32-5 ; 344-5-5$, and 344-9-5 lumps of undisturbed rock can be recognized in an extensively disturbed and mixed matrix (Figures 6-9). In other sections, for example, in 338-8-2, 338-22-5, 338-26-2, 339$6-2,341-6-2,341-31-2$, and $344-27-3$, it is questionable as to whether the observed inhomogeneities are natural or a consequence of mechanical disturbance (Figure 10). The photographs and core descriptions are provided so that the reader may reach his own conclusions.

\section{RESULTS AND DISCUSSION}

Numerical data for the samples are provided in Tables 2 and 3 . Table 1 contains the organic and carbonate carbon values for the individual lithologies recognized and sampled during the description of the split cores. In Table 2, the average carbonate carbon and organic carbon content of the core sections and the proportion of the organic fraction consisting of lipid (that is extractable oil?, and as kerogen (that is insoluble organic matter) are tabulated against geologic age and subbottom depth. Finally, Table 3 contains the carbon isotopic compositions of the lipid and kerogen fractions and the differences between these two values.

The geologically oldest samples on the $\mathrm{V}_{\phi}$ ring Plateau were obtained at Site 338. In age, the samples from this hole spanned the time interval from Pleistocene to early Eocene. This relatively broad time interval is present in a depth range of only 45.3 to 309 meters. These depths and the thermal histories, which 
TABLE 2

Carbonate Content and Concentrations (in wt \%) of Organic Fractions In Geochemical Samples from DSDP Leg 38

\begin{tabular}{|c|c|c|c|c|c|c|c|c|}
\hline \multirow{2}{*}{$\begin{array}{l}\text { Site-Core- } \\
\text { Section }\end{array}$} & \multirow{2}{*}{$\begin{array}{c}\text { Geologic } \\
\text { Age }\end{array}$} & \multirow{2}{*}{$\begin{array}{l}\text { Subbottom } \\
\text { Depth }(\mathrm{m})\end{array}$} & \multicolumn{2}{|c|}{ Carbonate ${ }^{\mathrm{a}}$} & \multicolumn{3}{|c|}{ Organic Carbon $^{\mathrm{a}}$} & \multirow{2}{*}{$\begin{array}{l}\text { Total } \\
\text { Lipid }^{b}\end{array}$} \\
\hline & & & Carbon & $\mathrm{CaCO}_{3}$ & Total & Lipid & Kerogen & \\
\hline $338-5-5$ & Pliocene or Pleistocene & $45.3-45.5$ & 0.26 & 2.17 & 0.49 & 0.005 & 0.49 & 1.0 \\
\hline $338-8-2$ & Middle Miocene & $78.8-79.0$ & 0.05 & 0.42 & 1.87 & 0.016 & 1.86 & 0.9 \\
\hline $338-11-2$ & Middle Miocene & $106.8-107.0$ & 0.05 & 0.42 & 0.75 & 0.011 & 0.74 & 1.5 \\
\hline $338-13-5$ & Lower Miocene & $130.8-131.0$ & 0.05 & 0.42 & 1.29 & 0.020 & 1.27 & 1.6 \\
\hline $338-17-5$ & Lower Miocene & $165.8-166.0$ & 0.04 & 0.33 & 1.38 & 0.028 & 1.35 & 2.0 \\
\hline $338-19-4$ & Middle Oligocene & $185.3-186.5$ & 0.05 & 0.42 & 0.63 & 0.005 & 0.62 & 1.0 \\
\hline $338-22-5$ & Middle Oligocene & $216.3-216.5$ & 3.80 & 31.67 & 0.19 & 0.002 & 0.19 & 1.1 \\
\hline $338-24-5$ & Middle Oligocene & $235.3-25.5$ & 0.06 & 0.5 & 0.10 & 0.010 & 0.09 & 10.0 \\
\hline $338-26-2$ & Upper Eocene & $249.8-250.0$ & 0.04 & 0.33 & 0.11 & 0.004 & 0.11 & 3.6 \\
\hline $338-30-5$ & Undated & 292.3-292.5 & 0.04 & 0.33 & 0.43 & 0.003 & 0.43 & 0.7 \\
\hline $338-32-3$ & Lower Eocene & $308.3-309.0$ & 0.20 & 1.67 & 0.44 & 0.009 & 0.43 & 2.0 \\
\hline $339-4-2$ & Pleistocene & $29.6-29.8$ & 1.27 & 10.58 & 0.60 & 0.015 & 0.59 & 2.5 \\
\hline $339-6-2$ & Pleistocene & $49.3-49.5$ & 0.78 & 6.50 & 0.97 & 0.016 & 0.95 & 1.7 \\
\hline $339-8-4$ & Pliocene or Pleistocene & $70.8-71.0$ & 0.20 & 1.67 & 0.33 & 0.005 & 0.33 & 1.5 \\
\hline \multirow[t]{2}{*}{$339-12-1$} & Lower or Middle & $103.0-103.2$ & 0.07 & 0.58 & 0.15 & 0.019 & 0.13 & 12.7 \\
\hline & Oligocene & $10.8-11.0$ & 0.36 & 7.17 & 0.29 & 0.007 & 0.28 & 2.4 \\
\hline $341-4-1$ & Pleistocene & $29.8-30.0$ & 0.41 & 3.42 & 0.59 & 0.008 & 0.58 & 1.4 \\
\hline $341-6-2$ & Pleistocene & $50.3-50.5$ & 0.75 & 6.25 & 0.61 & 0.012 & 0.60 & 2.0 \\
\hline $341-8-3$ & Pliocene or Pleistocene & $70.8-71.0$ & 0.65 & 5.42 & 0.64 & 0.007 & 0.63 & 1.1 \\
\hline $341-10-5$ & Pliocene or Pleistocene & $92.8-93.0$ & 1.03 & 8.58 & 0.45 & 0.023 & 0.43 & 5.1 \\
\hline $341-20-3$ & Pliocene or Pleistocene & $241.8-242.0$ & 1.54 & 12.83 & 0.31 & 0.005 & 0.31 & 1.6 \\
\hline $341-23-4$ & Pliocene or Pleistocene & $319.3-319.5$ & 0.14 & 1.17 & 0.47 & 0.008 & 0.46 & 1.7 \\
\hline $341.25-5$ & Pliocene or Pleistocene & $339.8-340.0$ & 0.04 & 0.33 & 1.22 & 0.014 & 1.21 & 1.1 \\
\hline $341.28-5$ & Middle Miocene & $396.8-397.0$ & 1.05 & 8.75 & 0.89 & 0.012 & 0.88 & 1.4 \\
\hline $341-30-5$ & Middle Miocene & $415.8-416.0$ & 0.11 & 0.92 & 1.14 & 0.020 & 1.12 & 1.8 \\
\hline $341-32-5$ & Middle Miocene & $434.8-435.0$ & 0.12 & 1.00 & 1.65 & 0.037 & 1.61 & 2.2 \\
\hline $341-34-5$ & Middle Miocene & $453.8-454.0$ & 0.04 & 0.33 & 1.76 & 0.031 & 1.73 & 1.8 \\
\hline $344-5-5$ & Pliocene or Pleistocene & $37.3-37.5$ & 0.51 & 4.25 & 0.71 & 0.021 & 0.69 & 3.0 \\
\hline $344-7-5$ & Undated & $56.3-56.5$ & 0.16 & 1.33 & 0.36 & 0.010 & 0.35 & 2.8 \\
\hline $344-9-5$ & Undated & $73.3-73.5$ & 0.17 & 1.42 & 0.44 & 0.006 & 0.43 & 1.4 \\
\hline $344-11-1$ & Undated & $88.2-88.4$ & 0.04 & 0.33 & 0.45 & 0.043 & 0.41 & 9.6 \\
\hline $344-15-4$ & Pliocene & $130.8-131.0$ & 0.22 & 1.83 & 1.02 & 0.021 & 1.00 & 2.1 \\
\hline $344-27-3$ & Pliocene & $243.3-243.5$ & 0.25 & 2.08 & 1.03 & 0.029 & 1.00 & 2.8 \\
\hline $344-31-2$ & Miocene or U. Pliocene & $317.8-318.0$ & 0.31 & 2.58 & 0.76 & 0.018 & 0.74 & 2.4 \\
\hline $346-1-3$ & Pleistocene & $4.5-4.7$ & 0.99 & 8.25 & 0.12 & 0.001 & 0.12 & 0.8 \\
\hline $346-10-4$ & Middle Miocene & $88.1-88.3$ & 0.07 & 0.58 & 1.04 & 0.015 & 1.03 & 1.4 \\
\hline $346-12-4$ & Undated & $107.1-107.3$ & 0.08 & 0.67 & 0.85 & 0.009 & 0.84 & 1.1 \\
\hline $346-17-2$ & Eocene & $151.8-152.0$ & 0.07 & 0.58 & 0.44 & 0.012 & 0.44 & 2.7 \\
\hline $348-19-5$ & Lower Miocene & $272.7-273.0$ & 0.07 & 0.58 & 0.32 & 0.004 & 0.32 & 1.3 \\
\hline $348-23-5$ & Lower Miocene & $329.6-329.8$ & 0.06 & 0.50 & 0.47 & 0.003 & 0.47 & 0.6 \\
\hline $348-25-5$ & Lower Miocene & $377.3-377.5$ & 0.05 & 0.42 & 0.63 & 0.007 & 0.62 & 1.1 \\
\hline $349-9-3$ & Lower Oligocene & $152.8-153.0$ & 0.09 & 0.75 & 0.61 & 0.004 & 0.61 & 0.7 \\
\hline
\end{tabular}

${ }^{\mathrm{a}} \mathrm{Wt} \%$ with respect to total core sample.

$\mathrm{b}_{\mathrm{Wt}} \%$ with respect to total organic carbon.

might be deduced from them, may be deceptive because of mud diapirism.

With the exception of one interval in the middle Oligocene, the gross mineral matrix of the cores from Site 338 is relatively uniform. Clay minerals and silica predominate in the mud-silt particle size range. The exception contained $31.7 \%$ carbonates as calcium carbonate. Pyrite also is present in this interval. Any catalytic effect of the mineral matrix on the alteration of the organic matter with time, therefore, should be relatively uniform.

Overall the properties of the sedimentary section appear favorable for genesis of significant quantities of petroleum. With the exception of three core sections in the middle Oligocene and two sections in the Eocene, the samples are high in organic matter. The essentially marine origin of the organic source material is indicated by the carbon isotopic compositions. The fine- grained nature of the inorganic matrix suggests that deposition occurred in quiet water with a minimum of abiogenic oxidation.

Petroleum genesis, however, is in a primary stage even in the lower Tertiary samples near the base of the hole. This conclusion is supported by the large negative $\Delta \delta^{13}$ values in the last column of Table 3 and the low values of the ratio of lipid (oil) to total organic matter, or kerogen shown in Table 2. An apparent exception is Core 24 , Section 5 . In this case, the organic content of the sample is extremely low. The high lipid values, therefore, may be a consequence of limiting experimental precision. The lack of development of petroleum, particularly liquid oil, in these rocks probably reflects a low temperature history.

Sites 339 and 341 to the east of the basement high on the $\mathrm{V} \phi$ ring Plateau provide the opportunity to observe the effect of increase in the thermal history at 


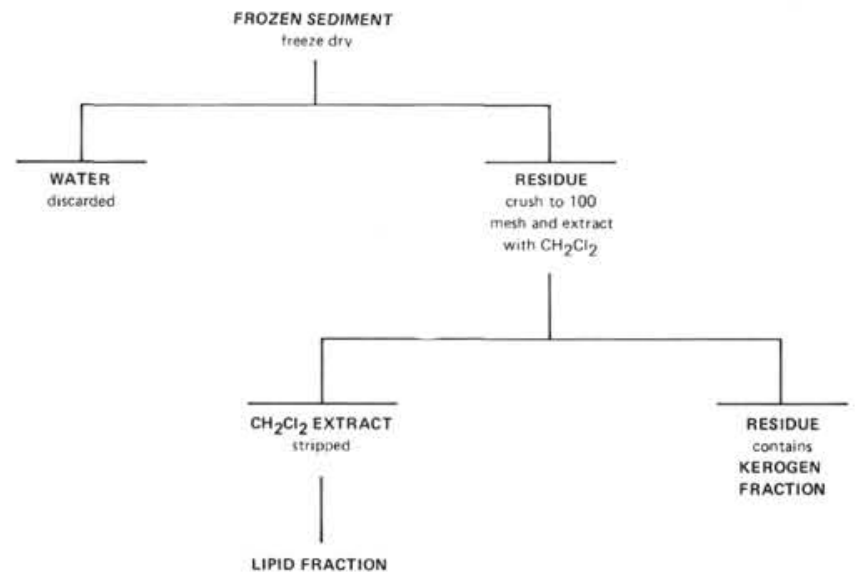

Figure 17. Flow diagram for separation and determination of carbon species.

equivalent age. The middle Oligocene core from near the bottom of the hole at Site 339 exhibits a $\triangle \delta C^{13}$ value of 0.1 and a high proportion of lipids (12.7\% of the total organic matter). These values are comparable to those for source rocks of many petroleum accumulations. The thermal history of this core, therefore, must have been much higher than would be suggested by the present depth of burial. At Site 341, petroleum genesis appears to be in a very early stage, probably as a consequence of a combination of a short depositional history (age) and low temperature as a consequence of shallow depth of burial. A possible exception is Core 10, Section 5 which shows a lipid (oil) to total organic ratio of $5 \%$. In this case, however, the heterogeneous particle size and mineral composition of the inorganic matrix suggests that the sedimentary history of the core as such may not be representative of the age and thermal history of the organic fraction.

The core sections from Sites 346, 348, and 349 closely resemble those from Site 338. For equivalent geologic age, the depths are shallow and the geothermal histories appear to have been low. Generally, the cores are far richer in organic matter than most marine sediments. Like their equivalent at Site 338 these sediments may have future petroleum potential, but at this stage in geologic time genesis is in a very early stage.

The suite of cores from Site 344 southwest of Spitzbergen, like most of those from Sites 346, 348, and 349 , are quite low in carbonate minerals. The organic content increases with an apparent trend to more marine conditions insofar as the source of the organic matter is concerned. Again the heterogeneous particle sizes of the mineral matrix suggest that at least some of the organic matter may be relict; hence, the high lipid to total organic value for Core 11, Section 1 at Site 344 may be the consequence of an age and thermal history quite different from the core itself.

\section{CONCLUSIONS}

Core samples from Sites 338, 339, and 341 on the V $\phi$ ring Plateau, from Sites 346,348 , and 349 to the west, and from Site 344 southwest of Spitzbergen are generally rich in organic matter derived from organisms which accomplished photosynthesis in a marine environment. Concentrations of carbonate minerals
TABLE 3

Carbon Isotopic Composition of Lipid and Kerogen Fractions in Geochemical Samples from DSDP Leg 38

\begin{tabular}{|c|c|c|c|}
\hline \multirow[b]{2}{*}{$\begin{array}{l}\text { Site-Core- } \\
\text { Section }\end{array}$} & \multicolumn{3}{|c|}{$\begin{array}{l}\text { Carbon Isotopic Composition, } \\
\qquad \delta \mathrm{C}_{\mathrm{PDB}}^{13} \\
\end{array}$} \\
\hline & Lipid & Kerogen & $\begin{array}{c}\text { Difference } \\
\text { L-K }\end{array}$ \\
\hline $338-5-5$ & -28.4 & -23.7 & -4.7 \\
\hline $338-8-2$ & -26.4 & -23.3 & -3.1 \\
\hline $338-11-2$ & -26.5 & -22.8 & -3.7 \\
\hline $338-13-5$ & -26.3 & -23.5 & -2.8 \\
\hline $338-17-5$ & -25.8 & -23.5 & -2.3 \\
\hline $338-19-4$ & -26.4 & -24.7 & -1.7 \\
\hline $338-22-5$ & -27.2 & -25.3 & -1.9 \\
\hline $338-24-5$ & -26.5 & -23.4 & -3.2 \\
\hline $338-26-2$ & - & - & - \\
\hline $338-30-5$ & -27.5 & -25.8 & -1.7 \\
\hline $338-32-3$ & -26.9 & -25.2 & -2.7 \\
\hline $339-4-2$ & -28.0 & -24.7 & -3.3 \\
\hline $339-6-2$ & -27.7 & -24.9 & -2.8 \\
\hline $339-8-4$ & -27.0 & -24.5 & -2.5 \\
\hline $339-12-1$ & -28.1 & -28.2 & -0.1 \\
\hline $341-2-1$ & -27.4 & -23.4 & -4.0 \\
\hline $341-4-1$ & -27.8 & -24.6 & -3.2 \\
\hline $341-6-2$ & -27.3 & -24.4 & -2.9 \\
\hline $341-8-3$ & -27.7 & -25.0 & -2.7 \\
\hline $341-10-5$ & -28.8 & -24.7 & -4.1 \\
\hline $341-20-3$ & -28.2 & -25.3 & -2.9 \\
\hline $341-23-4$ & -27.5 & -24.8 & -2.7 \\
\hline $341-25-5$ & -26.3 & -22.8 & -3.5 \\
\hline $341-28-5$ & -25.9 & -23.9 & -2.0 \\
\hline $341-30-5$ & -26.4 & -23.5 & -2.9 \\
\hline $341-32-5$ & -26.3 & -24.1 & -2.2 \\
\hline $341-34-5$ & -26.3 & -23.1 & -3.2 \\
\hline $344-5-5$ & -27.4 & -24.7 & -2.7 \\
\hline $344-7-5$ & -28.2 & -24.8 & -3.4 \\
\hline $344-9-5$ & -28.5 & -24.2 & -4.3 \\
\hline $344-11-1$ & -27.1 & -24.3 & -2.8 \\
\hline $344-15-4$ & -27.8 & -23.6 & -4.2 \\
\hline $344-27-3$ & -26.5 & -23.9 & -2.6 \\
\hline $344-31-2$ & -26.2 & -23.9 & -2.3 \\
\hline $346-1-3$ & -28.1 & -24.1 & -4.0 \\
\hline $346-10-4$ & -27.8 & -24.4 & -3.4 \\
\hline $346-12-4$ & -26.9 & -24.6 & -2.3 \\
\hline $346-17-2$ & -26.7 & -25.5 & -1.2 \\
\hline $348-19-5$ & -26.8 & -23.0 & -3.8 \\
\hline $348-23-5$ & -25.8 & -22.8 & -3.0 \\
\hline $348-25-5$ & -26.6 & -24.6 & -2.0 \\
\hline $349-9-3$ & -27.9 & -25.8 & -2.1 \\
\hline
\end{tabular}

decrease progressively to the west and north. Conditions thus far in geologic time appear favorable for petroleum genesis. With one exception, namely, Core 12 , Section 1 of lower to middle Oligocene age at Site 339 , petroleum genesis appears to be in a very early stage. The advanced stage of petroleum genesis in this core suggests that the lower to middle Oligocene at the location has been displaced upward, possibly by diapirism.

\section{REFERENCES}

Erdman, J.G., 1975a. Geochemical formation of oil. In Fischer, A.G. and Judson, S. (Eds.), Petroleum and global tectonics: Princeton University Press.

1975b. Time and temperature relations affecting the origin, expulsion and preservation of oil and gas: Paper 1, Panel Discussion 3, 9th World Petroleum Congr., Tokyo. 